\title{
BMJ Open Decisional support for young people who self-harm: protocol for a feasibility trial
}

\author{
Sarah L Rowe, ${ }^{1}$ Rebecca S French, ${ }^{2}$ Claire Henderson, ${ }^{1}$ Dennis Ougrin, ${ }^{3}$ \\ Mike Slade, ${ }^{4}$ Paul Moran ${ }^{5}$
}

To cite: Rowe SL, French RS, Henderson $\mathrm{C}$, et al. Decisional support for young people who self-harm: protocol for a feasibility trial. BMJ Open 2016;6:e012161. doi:10.1136/bmjopen-2016012161

- Prepublication history and additional material is available. To view please visit the journal (http://dx.doi.org/ 10.1136/bmjopen-2016012161).

Received 7 April 2016 Revised 6 June 2016 Accepted 4 August 2016

CrossMark

For numbered affiliations see end of article.

Correspondence to

Dr Sarah L Rowe;

sarah.rowe@kcl.ac.uk

\section{ABSTRACT}

Introduction: Self-harm is common in adolescents, and it is the strongest predictor of suicide. Young people who self-harm are often unsure of how and where to get help. Decision aids (DAs) have been shown to help with decisional conflict where there is uncertainty around different options. We have developed an online DA to support young people in help-seeking for self-harm. A feasibility trial will examine the acceptability of the online intervention and the ability to recruit and follow-up participants within a school setting.

Methods and analysis: In this parallel arm, singleblind feasibility trial, 60 participants aged 12-18 years who have self-harmed in the past 12 months, will be randomised to either (1) a group receiving the online DA or (2) a control group receiving general information about feelings and emotions. Both groups will complete measures assessing decision-making and help-seeking behaviour. The school counsellor will be notified of any participants who have been randomised to ensure safeguarding for the young person.

Participants in both groups will be followed up at 4 weeks, and the measures will be repeated. Qualitative interviews will be conducted with a subset of participants to explore their views and experiences of the DA and of participation in the study.

Ethics and dissemination: Ethical approval was granted by King's College London (KCL) College Research Ethics Committee. Results of this study will help to clarify if we can recruit and administer an online decisional support intervention within a school setting for young people who self-harm. The study will inform the design and implementation of a larger randomised controlled trial to test the effectiveness of the DA. Dissemination of the study findings will target publication in peer-reviewed journals of general and special interest. The funder will be sent a report outlining the major findings of the study.

Trial registration number: ISRCTN11230559.

\section{INTRODUCTION}

The term self-harm is used to describe a wide range of behaviours with a non-fatal outcome in which an individual deliberately

\section{Strengths and limitations of this study}

- We have developed an innovative personalised decision aid (DA) aimed at supporting young people in help-seeking for self-ham which is being tested in a school based population of young people aged 12-18 years.

- In addition to outcome measures examining decisional conflict, stage of decision-making, stigma and help-seeking, we will also conduct qualitative interviews that ask questions about participation in the study, thoughts on the intervention, safeguarding measures and recommendations for improvements to the DA or study.

- Because of the need to obtain parental consent, our ability to recruit a sufficient number of adolescents may be limited.

- Safeguarding procedures may act as a barrier to young people participation and enforce a helpseeking option.

- We are collecting limited information regarding the participants self-harm as the study is primarily focused on the impact the DA has on decision-making rather than decreasing future self-harm.

initiates behaviour (such as self-cutting) or ingests a substance, an illicit drug or noningestible substance or object, with the intention of causing harm to themselves. ${ }^{1}$

Self-harm is a serious health problem, and those who repeatedly self-harm can be left with permanent damage and disability. It is also a strong predictor of suicidal thoughts and behaviour ${ }^{2}$ and is especially prevalent in young people. ${ }^{3}$

Young people who self-harm often find it hard deciding where and how to get help, ${ }^{4}$ thus increasing their risk of sustaining serious medical harm. While young people may be reluctant to seek help from healthcare professionals when distressed, ${ }^{5}$ many turn to the internet for information or support as a way of coping with psychological distress. $^{6}{ }^{7}$ A growing number of trials 
involving children and adolescents suggest positive effects of interventions that make use of web-based technology. ${ }^{8}$ Therefore, a safe and effective, web-based intervention for young people who self-harm could be of benefit to large numbers of individuals.

Decision aids (DA) are designed to help individuals make a specific and deliberate choice between two or more options where there is uncertainty. They can be used in medical decision-making when individuals need to choose between treatment or screening options. ${ }^{9}$ A Cochrane review of 115 studies assessing the effects of DAs found that DAs produced higher knowledge, more active participation in decision-making and lower levels of decisional conflict. ${ }^{10}$ A DA may therefore be a useful intervention for young people who self-harm, to increase knowledge about help-seeking, reduce decisional conflict and increase active participation in decision-making when seeking help for self-harm.

In this article, we describe (1) the development of a novel, web-based DA, designed to support young people who self-harm, and (2) the methods of a study, which will investigate the feasibility of conducting a randomised controlled trial (RCT) of the DA.

\section{METHODS AND ANALYSIS \\ Overview}

This study is a two-group, parallel arm, single-blind, feasibility trial. Having obtained the prior consent of parents, we will invite young people, aged 12-18 years from a London secondary school, to complete a questionnaire about their mood and feelings and any selfharm behaviour they may have engaged in over the previous year. Participants who indicate they have selfharmed in the previous year will then be randomised to either the group receiving the DA or a control group who will receive general information about feelings and emotions. Both groups will complete outcome measures on decision-making, intentional and actual help-seeking behaviour. Participants in both groups will be followed up at 4 weeks when we will repeat the scales. Qualitative interviews will be conducted to explore young people's views and experience of participation in the study and, for those in the intervention group, of the DA.

\section{Aims and objectives}

1. To assess the feasibility of undertaking a RCT of the online DA, with respect to the following predefined parameters:

- recruitment of young people and consent rates;

- the feasibility and acceptability of randomisation procedures;

- follow-up rates and response rates to questionnaires;

- feasibility and acceptability of the intervention delivered in a school setting.
2. To report descriptive data on candidate outcome measures, in order to inform the design of an adequately powered, future effectiveness study.

\section{Eligibility criteria}

Inclusion criteria

1. Aged $12-18$ years

2. Attending the study site, a secondary school within an inner London borough

3. Basic proficiency in English language

4. Self-harmed in the past 12 months

\section{Exclusion criteria}

1. Lacking capacity to consent

2. Poor English language proficiency

3. Episodes of self-harm not in the past 12 months

\section{Measures}

\section{Baseline assessments: all participants}

1. Socio-demographic schedule: This includes questions on gender, age, ethnicity, who they live with and whether or not the participants have a sibling, boyfriend/girlfriend or social worker. Responses to these questions are relevant to the options available for users to select in the DA (eg, if a user does not have a boyfriend/girlfriend, this help-seeking option will not be listed) and to describe the sample.

2. Short Mood and Feelings Questionnaire (SMFQ): This assessment screens for depression among children aged 16 and under, ${ }^{11}$ and its use has been validated in late adolescence. ${ }^{12}$ It consists of 13 statements that are reflective of depressive symptoms. Ratings are given on a 3-point scale, ranging from 'true', 'sometimes' and 'not true', with 'true' indicating that the depressive symptom is present. 'True' ratings are scored as 2, 'sometimes' as 1 and 'not true' as 0 . Total scores range from 0 to 26 , with a cut-off score of $>8$ indicating the likely presence of major depression.

3. Questions on self-harming behaviour: (1) 'Have you ever deliberately tried to harm yourself (such as cut yourself or taken an overdose)? (2) When was the last time you tried to harm yourself?

\section{DA group and control intervention}

4. Stage of Decision-Making Scale: This scale measures the individual's readiness to engage in decision-making. ${ }^{13}$ It consists of 1 item with 6 response options anchored at 1 (haven't started to think about the choices) and 6 (have already made a decision and am unlikely to change my mind).

5. General Help-Seeking Questionnaire (intentions) (GHSQ). This assesses future help-seeking intentions and recent and past help-seeking experiences. It uses a 7-point Likert scale ranging from 1 (extremely unlikely) to 7 (extremely likely) for each help source option. Help sources assessed are informal (eg, friends and family) and formal (eg, mental health 
professional and teacher). Higher scores indicated higher intentions. This scale has been tested on high school students aged 12-19 years and has satisfactory reliability and validity ( $\alpha$ range of $0.70-0.88) .{ }^{14}$

6. Questionnaire on Anticipated Discrimination (QUAD). This is a 14-item measure that has expanded on previous versions of the Discrimination and Stigma Scale. ${ }^{15}$ It measures the extent to which people anticipate personally experiencing discrimination in key life domains as a result of mental health problems. It uses a 4-point Likert scale ranging from $0=$ strongly disagree to $3=$ strongly agree. It has good internal consistency $(\alpha=0.86)$ and adequate convergent validity. ${ }^{16}$ We are using five items from this measure that are relevant to our study population (adolescents).

7. Decisional Conflict Scale: The decisional conflict scales measures personal perceptions of

- Uncertainty in choosing between options;

- Modifiable factors contributing to uncertainty including feeling uniformed, lack of clarity about personal values and feeling unsupported in decision-making;

- Effective decision-making such as feeling the choice is informed, values-based, likely to be implemented and expressing satisfaction with the choice.

The 16 -item version of the scale is the most commonly used. ${ }^{17}$ Each item is rated on a 5-point Likert scale ranging from $0=$ strongly agree to $4=$ strongly disagree. A total score and five subscores (uncertainty; informed subscore; vales clarity subscore; support subscore and effective decision subscore) are generated. Scores are generated by summing the items, dividing by the number of items in the (sub) scale and multiplying by 25 . Scores range from 0 (no decisional conflict) to 100 (extremely high decisional conflict). The scale has demonstrated adequate test-retest reliability. ${ }^{18}$ Scores exceeding 37.5 are associated with decisional delay or feeling unsure about implementation. We are asking questions pertaining to the support and uncertainty subscales only.

8. Questions on the DA

Participants will be asked to provide comments about (1) whether they would follow the 'recommended' option, (2) whether the use of the DA has changed any of their perceptions/feelings about the paths they can go down, (3) whether there is anything we could do to improve the DA and (4) whether or not they would recommend the DA to other young people who have self-harmed.

The support and uncertainty subscales from the Decisional Conflict Scale and the Stage of Decision-Making Scale will be repeated immediately after completing the DA.

\section{Follow-up assessment}

At 4 weeks, participants in the control group and the DA group will be asked to repeat an online assessment of the measures below:

1. GHSQ (actual)
2. Stage of Decision-Making Scale

3. QUAD

4. Decisional Conflict Scale

5. Questions on their experience of using DA

Follow-up assessments will take place at the school in the participant's lunch break or after school. Once this assessment is complete, they will be invited to participate in a qualitative interview, which will include exploration of factors relating to involvement in the study (eg, influences on participation, potential sources of contamination between control and intervention groups) and, for intervention participants, views and experiences of the DA (eg, how, if at all, the DA prompted help-seeking behaviour).

\section{Intervention}

\section{Development of the DA}

The theoretical underpinning of the DA developed for young people who self-harm is subjective utility analysis. The assumption here is that the decision-maker is provided with all the information to make a decision, including knowledge of the alternatives, and is aware of their values and preferences with regard each of these options. ${ }^{1920}$ In order to inform the development of the DA, we conducted a systematic literature review to identify help-seeking options and attributes that young people rate as important when considering where to go for support. ${ }^{21}$ In developing the DA, we conducted extensive consultation with health professionals and groups of young people. Qualitative interviews were conducted with young people in child and adolescent mental health services $(\mathrm{N}=5)$, adolescents recruited from a local school $(\mathrm{N}=6)$, ex-service users from ChildLine ( $\mathrm{N}=2$ ) and professional staff (including medics, a clinical psychologist and school counsellor, $\mathrm{N}=5$ ). Young people who participated in the qualitative interviews were then invited back and to complete the DA in order to observe the ease, acceptability and potential utility of the DA.

We then applied this evidence in the subsequent development of the DA. The online DA (termed My Self-Help Tool) was built using two pieces of proprietary software, Annalisa $\bigodot^{22}$ and Elicia $\odot .^{23}$ Annalisa is a multicriteria DA with a single screen interface that allows the user to create and explore decision-making scenarios. Elicia is a general purpose web-based questionnaire builder. Annalisa is embedded within Elicia, which allows the decision-making process to be customised and personalised according to questionnaire responses. Annalisa uses a direct weighting method, which is simpler and less time-consuming to use than other multicriteria methods, such as the analytic hierarchy process. ${ }^{24}$ My Self-Help Tool is designed for young people to be used by themselves, to find out about different help-seeking options for self-harm (such as family, General Practitioner (GP) and telephone helpline). These options were identified from the help-seeking literature and findings from qualitative interviews involving 


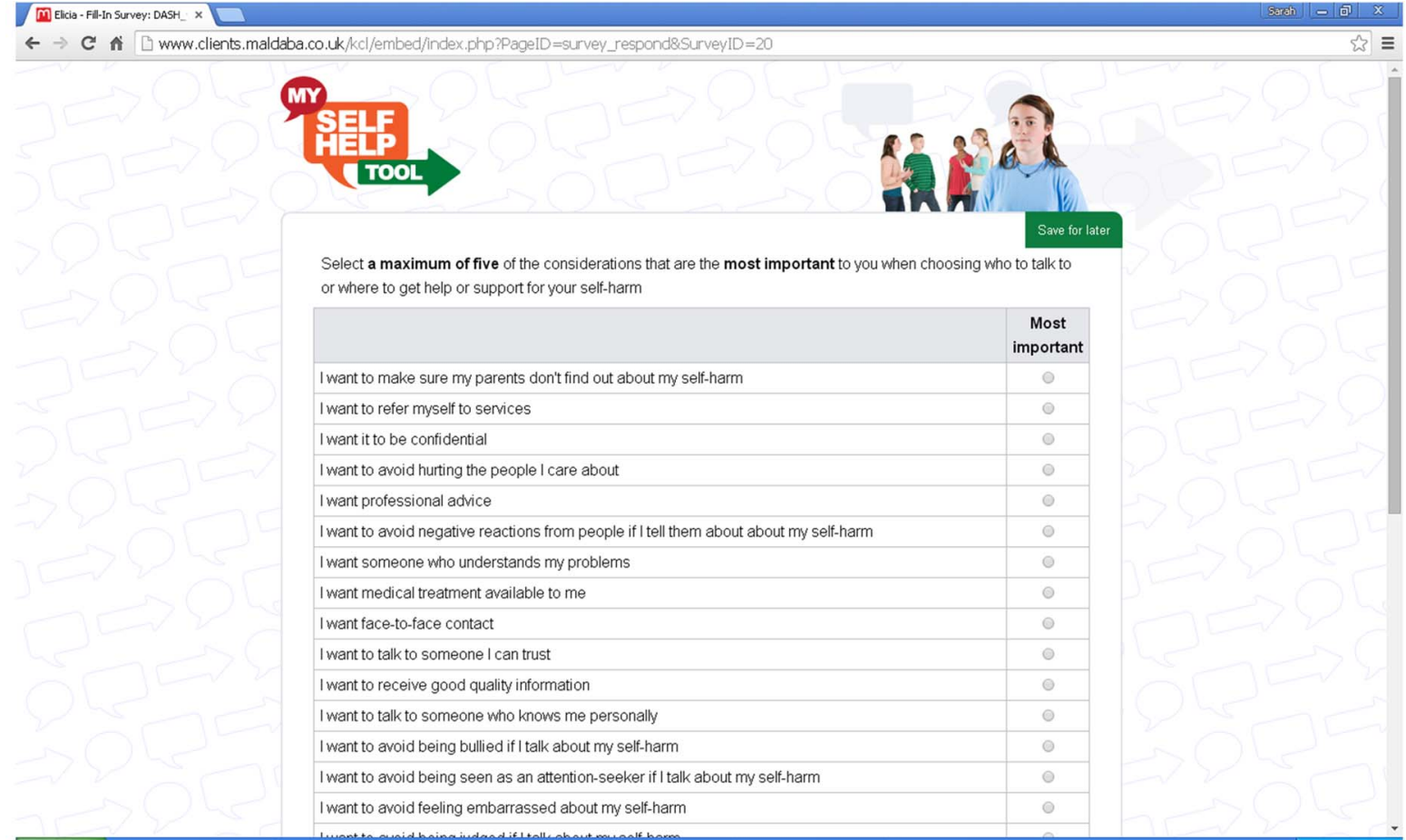

Annalisa (C) copyright Maldaba Ltd.

Figure 1 Help-seeking attributes.

young people and service providers. In addition to the sources of support, users are asked to identify helpseeking attributes that are important to them, ranging from confidentiality to other concerns (such as not wanting to be seen as attention seeking) (figure 1). They are then required to indicate the degree of importance they attach to each attribute they have identified according to the help-seeking options they have chosen, for example, weighting how important maintenance of confidentiality is to them. Weights are assigned through simple manipulation of a cursor on a single graphic screen that presents all the elements of a decision (figure 2); while participants visually assign weights, this is converted from 0 (not at all important) to 1 (extremely important) within the computer programme. As we are interested in the relative weights placed on attributes, these are normalised so that the total sum of weights equals 1 (or 100\%). Once they have made their selections, a personalised rating and ranking of the helpseeking options are presented to them, based on the information they have submitted. ${ }^{25}$ This is calculated in the underlying decision analysis $[\mathrm{w} 1+\mathrm{w} 2+\mathrm{w} 3 \mathrm{~L}] \times[\mathrm{p} 1+\mathrm{p} 2$ $+\mathrm{p} 3 \mathrm{~L}]=\mathrm{SCORE}$ ); the highlighted bar is the suggested option based on the individual user response (figure 3). Information on how to access help-seeking options is also provided. 'My Self-Help Tool' was constructed in consultation with a group of young people $(n=6)$, ex-service users $(n=2)$, young people currently accessing mental health services $(n=5)$ and health professionals $(n=5)$. These key stakeholder groups were asked to identify any missing additional support options and criteria that they considered important to help-seeking (attributes). We also obtained the stakeholders' advice on the wording used within the DA.

Panel groups of young people recruited from a local school, child and adolescent mental health services and Childline $^{\mathrm{i}}$ were invited to complete the DA to test progression through the DA and appropriateness of language.

\section{Control intervention}

Participants in the control arm will receive general information on feelings and emotions from the ChildLine website (http://www.childline.org.uk/Explore/FeelngsEmotions/ Pags/FeelingsEmotions.aspx). This information has been displayed as a static (non-interactive) page in our questionnaire rather than a link that young people can use to connect to the ChildLine website. This also promotes the young person's awareness of the ChildLine website, so that they can access the rest of the site after ratings have been completed. We have chosen this comparison group, so that we can assess if the DA effects decision-making over-and-above general information that is already available to young people on the internet.

${ }^{\mathrm{i}}$ ChildLine is a free online or telephone counselling service for children and young people up to 19 years of age, provided by the National Society for the Prevention of Cruelty to Children (NSPCC), a children's charity in the United Kingdom. Permission to use the general information on feelings and emotions from the ChildLine website was obtained from the ChildLine organisation. 


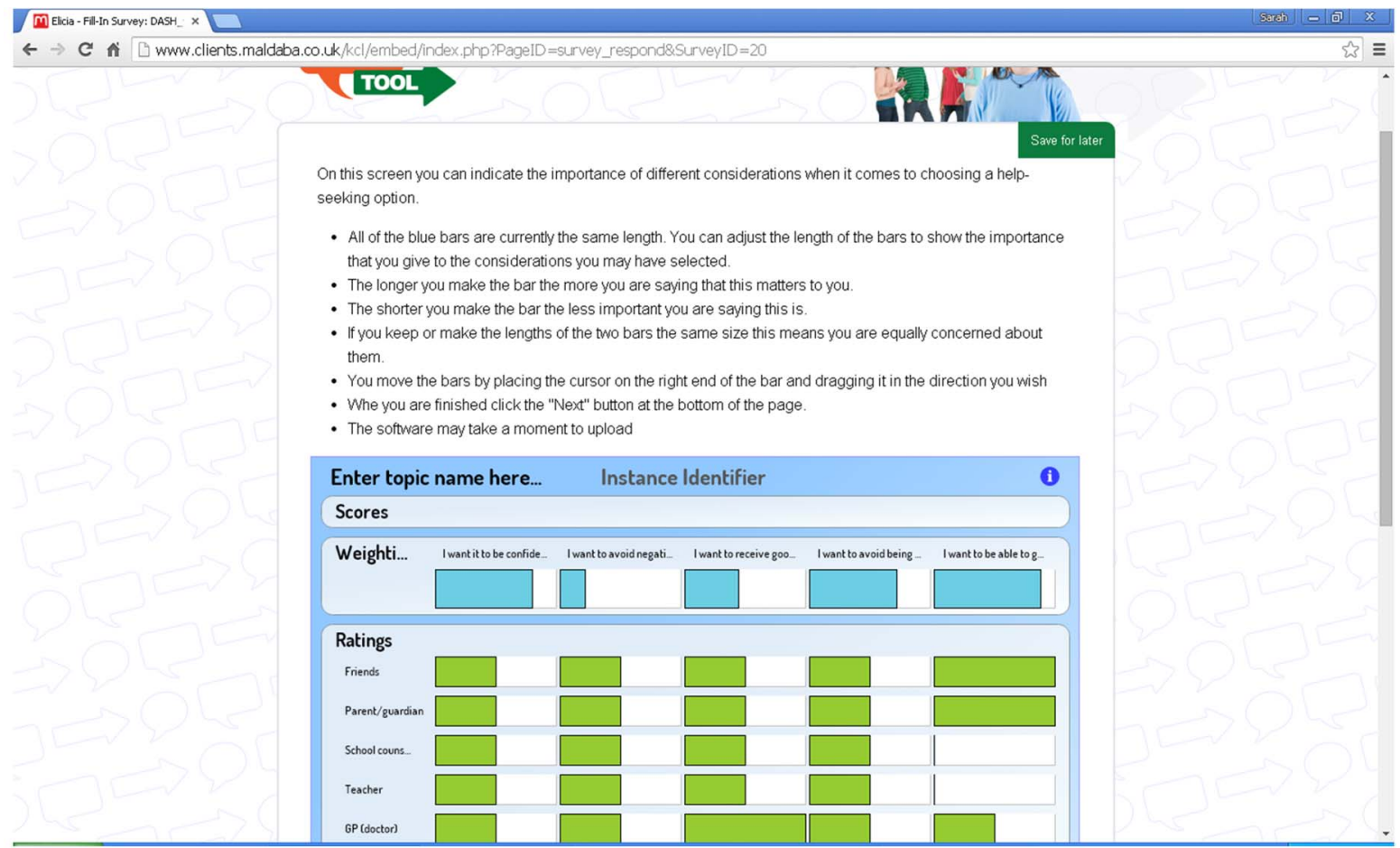

Annalisa (C) copyright Maldaba Ltd.

Figure 2 Weighting of attributes.

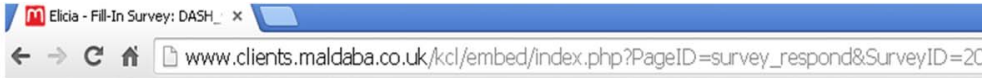

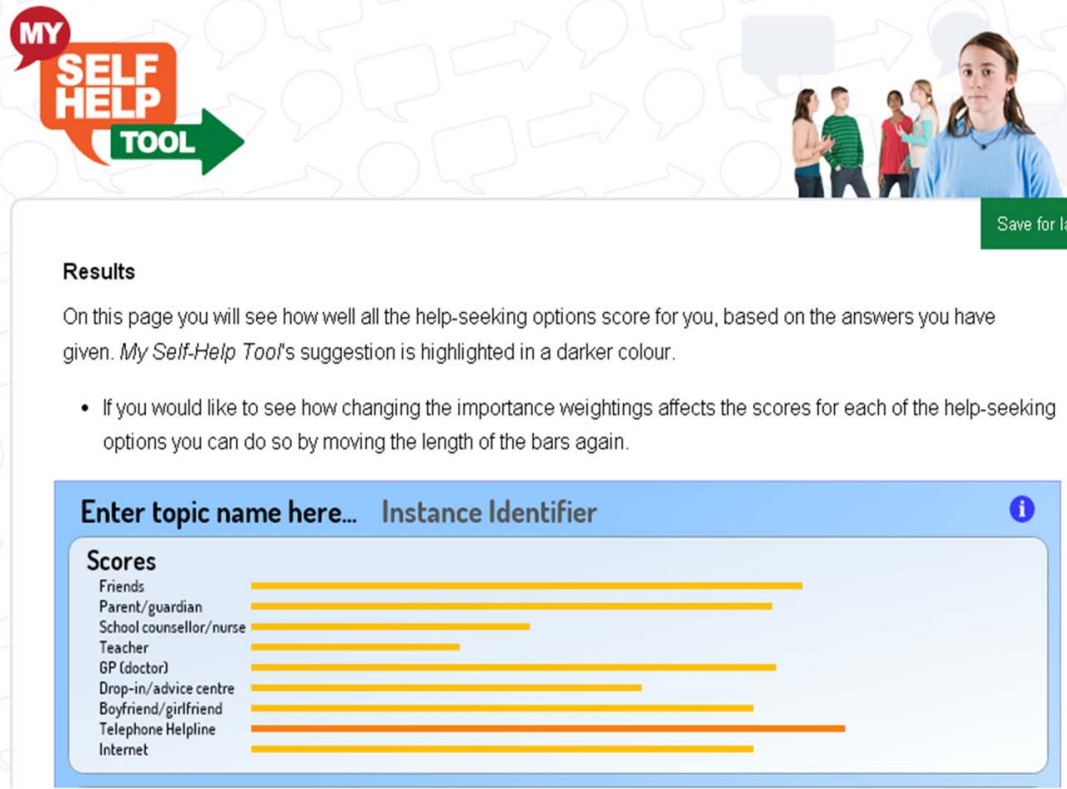

Annalisa (C) copyright Maldaba Ltd.

Figure 3 Suggested help-seeking option.

Recruitment and baseline procedures

Participants will be identified through a secondary school in an inner London borough (figure 4). All parents/guardians will be sent a letter, information sheet and consent form via post or email, asking for their consent to invite their child/children aged 1218 years to participate in the study. A range of dissemination activities has been undertaken to promote 


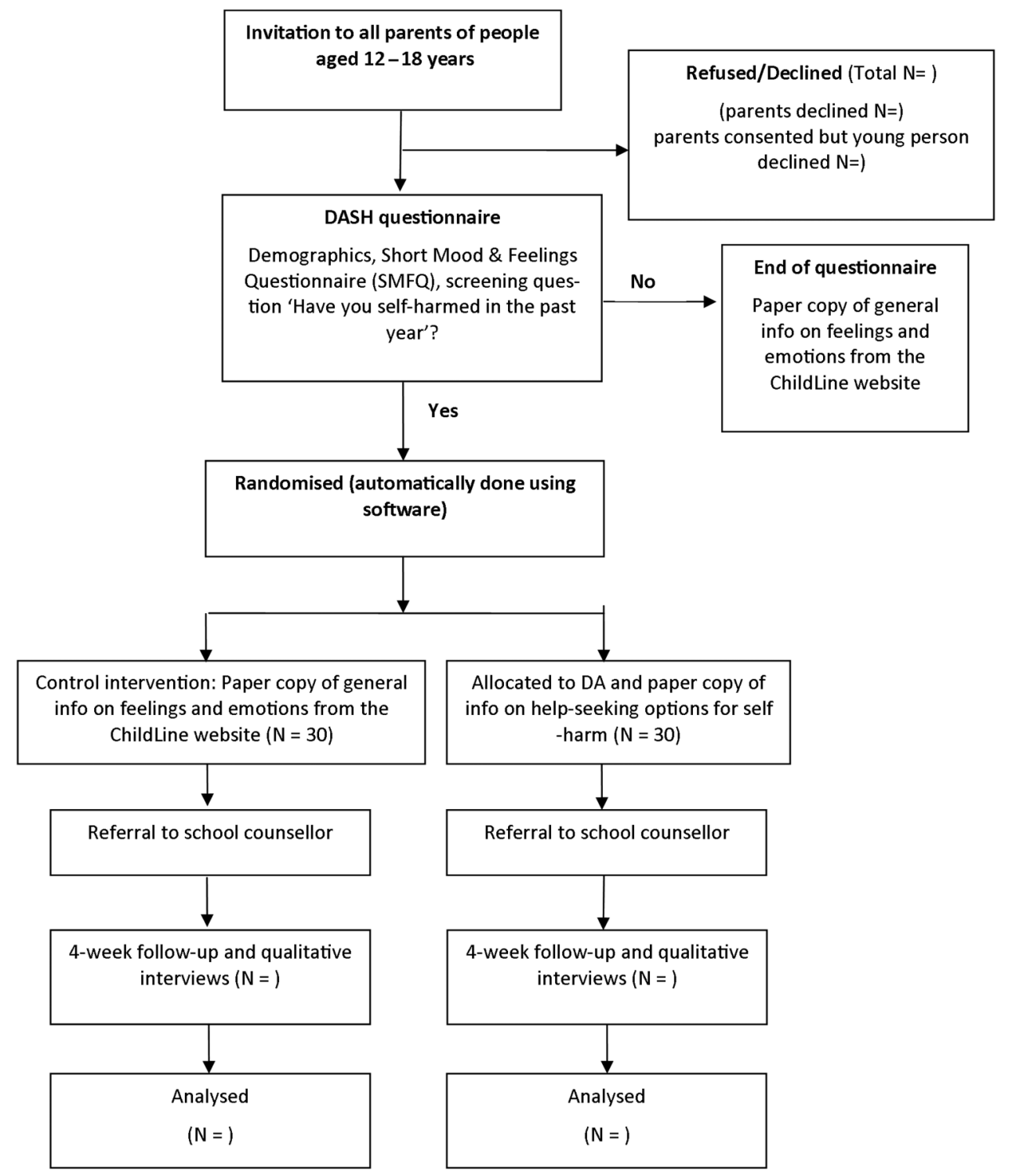

Figure 4 Flow chart of trial design. DAs, decision aids; SMFQ, Short Mood and Feelings Questionnaire.

awareness of the study among pupils and parents including presentations at school assemblies, school newsletters and circulation of a link to an audio recording of the research team providing information about the study. If consent is given, their child/children will be contacted by a member of the research team and invited to participate in the study. At an initial meeting with the young person, an information sheet and consent form will be provided and they will be given at least 24 hours in which to think about whether or not they wish to participate.

The young person will be contacted again and scheduled to complete the assessment. At the start of the appointment, the researcher will explain the study and the potential participant will be given the opportunity to ask the researcher questions. At the end of the explanation, the researcher will carefully assess the participant's understanding of the study and the consequences of participation by asking some questions regarding specific aspects of the study procedure such as 'What will your participation involve?' If satisfied that the participant understands the requirements, the researcher will invite the participant to sign the assent/consent form. Young people aged 12-15 will assent to participate, and 16-18 years old will sign consent forms.

The young person's information sheet and consent form have been developed in consultation with adolescents aged 12-16 years old, so that the language and information provided are age-appropriate (see online supplementary appendices A and B, respectively).

Once consent has been obtained, the young person will complete an online questionnaire at school in their lunch break or after school, asking demographic questions (eg, age and gender), a short questionnaire about their mood and feelings and a question about any selfharm behaviour in the previous year. If the participant has not self-harmed in the previous year (this includes those who have never self-harmed and those who self- 
harmed more than 12 months ago), then the questionnaire will end and they will be given a paper copy of general information about feelings and emotions from the ChildLine website. Completion of the questionnaire in this instance is a onetime only occurrence that will take $\sim 5-10 \mathrm{~min}$. All participants will receive a $£ 5$ voucher on completion of the assessment to thank them for their time and trouble.

If the participant reports that they have self-harmed in the previous 12 months, they are automatically randomised by the computer programme to one of two groups: (1) a DA group whereby they complete the DA and are presented with help-seeking options based on information they have provided while using the DA or (2) a control group whereby they are given general information about feelings and emotions from the ChildLine website. Once they have completed the DA, they receive a paper copy of information on how to access any of the help-seeking options that are listed in the DA. Participant allocation to the group receiving the DA or the control group will be subject to simple randomisation, which will be conducted remotely.

All randomised participants will complete baseline measures before and after they go through the DA or read the general ChildLine information.

The process of consent and the administration of the measures and DA were tested on five young people aged 12-16 years. On average, consent and completion of the measures and DA took 10-15 min, with no dropouts.

\section{Randomisation}

Participants who are randomised into the experimental groups (ie, those who have self-harmed in the past year) will be placed into one of eight trial strata (all boys will be grouped into a single stratum, and girls will be grouped into seven age strata). We are stratifying randomisation by gender because self-harm typically occurs more frequently in female adolescents compared to male adolescents. ${ }^{26}$ Each stratum will be randomised using the random permuted block algorithm, with a block size of four. Appropriate locks are in place to ensure that it will not be possible for a randomisation token to be used multiple times or skipped over.

\section{Safety protocols}

All participants who disclose self-harm during the study (irrespective if it is in the past 12 months or more than 12 months ago) will be referred to the school counsellor in order to ensure that they remain safe and are given appropriate support. As per usual school policy, the school will notify parents if their child has been referred to the school counsellor. Participants may be concerned about their self-harm being disclosed to the school counsellor and their parents, however, this protocol will be clearly stated in the information sheet and consent form and discussed with the researcher so that potential participants are aware of this before agreeing to participate.
A Trial Steering Committee (TSC) has been formed in order to monitor progress of the trial adherence to the protocol, to ensure the safety and well-being of the trial participants are the most important considerations and prevail over the interests of the science and society. The TSC includes a student representative from the participating school.

\section{Sample size}

Sample size calculations are not required for most feasibility studies, because the aim is to gather information about recruitment processes, consent and attrition rates and trial procedures. Nevertheless, we want to know whether it is feasible to recruit and retain a predetermined number of young people who self-harm into a trial of the DA- a group of young people who may be more difficult to recruit into a trial. For this reason, we have undertaken a power calculation, in order to give us a target number to aim towards. For continuous outcomes relating to decisionmaking and empowerment, a sample size of 60 would detect a standardised effect size of 0.75 , with $80 \%$ power. The RCT will also allow us to measure any differences in helpseeking behaviour associated with use of the DA. Assuming a help-seeking prevalence of $20 \%,{ }^{27}$ a sample size of 60 (30 randomised to the DA, 30 randomised to the control condition) will have greater than $80 \%$ power to detect a threefold increase in categorically defined help-seeking behaviour (0.20 to 0.60$)$ based on a twosided $\chi^{2}$ test at $5 \%$ significance.

The 1-year prevalence of self-harm in school children has been found to be $\sim 10 \%{ }^{1}$ In order to obtain a sample of 60 young people reporting self-harm, we will therefore need to recruit 600 pupils. For 540 of these pupils, their participation will simply involve completion of some short demographic measures, the SMFQ and a question about their self-harm. The remaining 60 pupils will be randomised to receive either the DA, or the control intervention.

\section{Statistical analysis plan}

In keeping with recommendations for small-scale feasibility trials, the analysis will focus on feasibility of scaling up to a full-scale RCT. In this study, it will consist of the following:

1. To determine feasibility of recruitment to the study, all referrals to the study will be documented, and the number of referrals meeting inclusion/exclusion criteria will be examined. Details of those who decline to be randomised and an option for their reason for refusing will be retained.

2. Treatment acceptability will be assessed by the number of participants who refuse to use the DA. Retention up until 4-week follow-up will be examined.

3. Feasibility of the research protocol will be assessed by the number of participants failing to adhere to the full research protocol, the burden of which will be similar to that which could be expected in a full 
study. The target would be collection of complete data on $90 \%$ of all service users recruited.

4. We will report exploratory findings of the outcome measures and use a confidence intervals approach to assist the justification for proceeding to a phase III trial.

\section{Process evaluation}

Qualitative interviews will be conducted on a subgroup of participants to explore their views and experiences of participating in the study, and, for those in the intervention group, of the DA, including its acceptability and (potential) effects. All participants who have been randomised into either the intervention group or the control group will be invited for individual interviews, which will take place at the school with a member of the research team, shortly after the 4-week follow-up. It will include questions on factors that may have influenced their participation in the study, thoughts on the intervention, following the advice of the DA, confidentiality, safeguarding measures (ie, the referral to the school counsellor) and recommendations for improvements to the DA or the study. We anticipate involving 5-10 young people in each of the experimental groups and 3-5 young people from the excluded group (the group that were not randomised to the DA or the control intervention). With consent, we will audio record the interviews and transcribe these so they can be analysed using thematic analysis. Interview transcripts will be repeatedly examined in depth and coded in reference to the experience of participating in the DASH study; this iterative method of constant comparison will be used in order to reduce and condense themes into the most salient categories. Subordinate themes will be established for each transcript, and then cross-validated, adjusting if required. Superordinate themes will then be chosen on the basis of forming 'umbrella' themes that cover various subordinate themes. Data will be analysed by two researchers; joint discussions will be held to ensure that codes and themes are adequately grounded in the raw data.

\section{DISCUSSION}

Web-based interventions have been associated with increased mental health literacy and reduced levels of stigma connected to help-seeking (refs. 27-29 in ref. 30). However, testing the usability and potential efficacy of a web-based DA for young people who self-harm is not without its challenges and limitations. There are two main challenges with conducting research in this area: first, there is the issue of consent. In the UK, young people under the age of 16 years require parental consent. Although young people aged 16 and above can consent for themselves, it is best practice to obtain parental consent prior to their involvement in any research. This creates a potential barrier for recruitment with some evidence showing that response rates are lowered by $40-67 \%$ where active parental consent is required, resulting in lower participation in school surveys by at-risk groups. ${ }^{32}$ We aim to address this by engaging with the parents using various recruitment methods, for example, post, email, school newsletter and audio recording. Second, the disclosure of self-harm in an adolescent population necessitates the need for robust safeguarding procedures in order to ensure that if a young person is at further risk of harm, they are provided with appropriate support. However, the process of informing the school counsellor (and potentially the parents) may conceivably result in the young person not disclosing any issues with self-harm they are experiencing; subsequently alienating the very people this DA may benefit. We will try to capture information regarding reasons for non-disclosure or not participating during the process evaluation, in order to detect any impact of safeguarding protocols on participation.

Our safeguarding protocol may further act as a study limitation, as we are enforcing a help-seeking option after completion of the baseline assessment by informing the school counsellor of their self-harm. At the 4-week follow-up, the GHSQ (actual) asks whether or not they have sought help for their self-harm from a variety of different sources, for example, parents/carer, friends, doctor, school counsellor, etc. From this (and information from the school counsellor), we will be able to differentiate our safeguarding procedures from other actual help-seeking options that may have been sought since their baseline assessment.

The feasibility trial will allow us to test if we can recruit and administer a decisional support intervention for young people who self-harm within a school setting. When we originally developed the DA, it was with the intention that this would be an online tool that is available to all young people who self-harm. However, we would be unable to provide support and safeguarding for adolescents at risk of harm. This limits the generalisability of our study, and any future study should consider how this tool could made more widely available while providing support for young people at risk.

A final consideration in our study is the limited information we are collecting regarding the participant's selfharm. We have chosen to only focus on the presence/ absence of self-harm in the previous 12 months rather than obtaining details on frequency, type and severity simply because the study is primarily concerned with the effect that the DA has on decision-making, rather than any effect it may have on decreasing future self-harm.

Despite the limitations and challenges of this study, we have developed an innovative personalised DA aimed at helping young people who are engaging in risky behaviour and where they may spuriously perceive their support options to be limited. Depending on the outcome of this feasibility study, there is scope to conduct a larger RCT evaluating the DA or to broaden the DA so it covers help-seeking options for general mental health issues and resilience.

\section{DISSEMINATION}

The results of the research will be targeted for publication in peer-reviewed journals of general and special interest. At the conclusion of the trial, the funder will be sent a report 
outlining the major findings of the study. We will also produce a short report of the main study findings for all participants. The results from the trial will be analysed and presented first to a meeting to the TSC for comment and discussion. Results will subsequently be published as soon as possible under the authorship of the writing committee. The writing committee will consist of the Principal Investigator, the postdoctoral research worker and members of the Project Advisory Group. All publications that result from the trial shall include a list of members of the TSC. The ISRCTN number will be attached to any publications from this trial. Funding from Guy's and St Thomas' Charity and Childline support will be acknowledged in any resulting publication.

\section{Author affiliations}

${ }^{1}$ Health Service and Population Research Department, King's College London, Institute of Psychiatry, Psychology and Neuroscience, London, UK

${ }^{2}$ Department of Social and Environmental Health Research, Faculty of Public Health \& Policy, London School of Hygiene and Tropical Medicine, London, UK ${ }^{3}$ Child and Adolescent Psychiatry Department, King's College London, Institute of Psychiatry, Psychology and Neuroscience, London, UK

${ }^{4}$ School of Health Sciences, Institute of Mental Health, University of Nottingham, Nottingham, UK

${ }^{5}$ Centre for Academic Mental Health, School of Social \& Community Medicine, University of Bristol, Bristol, UK

Acknowledgements The authors express their gratitude to Lorenzo Gordon and Mark Clements from Maldaba who provided the Annalisa and Elicia software used for the decision aid (DA), randomisation, support and advice regarding the development of the DA and study methodology. The authors thank Sue Minto and David Weatherall, members of ChildLine, for their contributions to the project management group and methodological advice. They also especially thank the students and staff who helped with the development and piloting of the DA.

Contributors SLR helped to develop the decision aid, involved in the study design and drafted the paper. RSF and PM participated in the development of the decision aid, study design and commented on the draft of the paper. $\mathrm{CH}, \mathrm{DO}$ and MS involved in the study design and commented on the draft of the paper.

Funding The DASH study is funded by Guy's and St Thomas' charity (project code: MAJ120701).

Competing interests None declared.

Patient consent Obtained.

Ethics approval The feasibility trial was approved by King's College London (KCL) College Research Ethics Committee (CREC); reference number PNM/14/ 15-114.

Provenance and peer review Not commissioned; externally peer reviewed.

Open Access This is an Open Access article distributed in accordance with the Creative Commons Attribution Non Commercial (CC BY-NC 4.0) license, which permits others to distribute, remix, adapt, build upon this work noncommercially, and license their derivative works on different terms, provided the original work is properly cited and the use is non-commercial. See: http:// creativecommons.org/licenses/by-nc/4.0/

\section{REFERENCES}

1. Madge $\mathrm{N}$, Hewitt $\mathrm{A}$, Hawton $\mathrm{K}$, et al. Deliberate self-harm within an international community sample of young people: comparative findings from the Child \& Adolescent Self-harm in Europe (CASE) Study. J Child Psychol Psyc 2008;49:667-77

2. Whitlock J, Muehlenkamp J, Eckenrode J, et al. Nonsuicidal self-injury as a gateway to suicide in young adults. $J$ Adolesc Health 2013;52:486-92.
3. Muehlenkamp JJ, Claes L, Havertape L, et al. International prevalence of adolescent non-suicidal self-injury and deliberate self-harm. Child Adolesc Psychiatry Ment Health 2012;6:10.

4. De Leo D, Heller TS. Who are the kids who self-harm? An Australian self-report school survey. Med J Aust 2004;181:140-4.

5. Fortune S, Sinclair J, Hawton K. Help-seeking before and after episodes of self-harm: a descriptive study in school pupils in England. BMC Public Health 2008;8:369.

6. Baker D, Fortune S. Understanding self-harm and suicide websites: a qualitative interview study of young adult website users. Crisis 2008;29:118-22.

7. Jones R, Sharkey S, Ford T, et al. Online discussion forums for young people who self-harm: user views. Psychiatrist 2011;35:364-8.

8. Lau EY, Wong DP, Ransdell L. A systematic review of information and communication technology-based interventions for promoting physical activity behavior change in children and adolescents. $J$ Med Internet Res 2011;13:4-4.

9. An introduction to patient decision aids. BMJ 2013;347:f4147.

10. Stacey D, Légaré F, Col NF, et al. Decision aids for people facing health treatment or screening decisions. Cochrane Database Syst Rev 2014;(1):CD001431.

11. Angold A, Costello EJ, Messer SC, et al. Development of a short questionnaire for use in epidemiological studies of depression in children and adolescents. Int J Meth Psych Res 1995;5:237-49.

12. Turner N, Joinson C, Peters TJ, et al. Validity of the Short Mood and Feelings Questionnaire in late adolescence. Psychol Assessment 2014;26:752-62

13. O'Connor A. User manual-stage of decision making. Secondary user manual—stage of decision making. 2000. http://www.ohri ca/decisionaid

14. Wilson CJ, Deane FP, Ciarrochi JV, et al. Measuring help seeking intentions: properties of the general help seeking questionnaire. Can $J$ Couns 2005;39:15-28.

15. Brohan E, Clement S, Rose D, et al. Development and psychometric evaluation of the Discrimination and Stigma Scale (DISC). Psychiat Res 2013;208:33-40.

16. Gabbidon J, Brohan E, Clement S, et al. The development and validation of the Questionnaire on Anticipated Discrimination (QUAD). BMC Psychiatry 2013;13:297.

17. O'Connor A. User manual—Decisional Conflict Scale. 1993 (updated 2010). www.ohri.ca/decisionaid

18. O'Connor AM. Validation of a decisional conflict scale. Med Decis Making 1995;15:25-30.

19. Bekker H, Thornton JG, Airey CM, et al. Informed decision making: an annotated bibliography and systematic review. Health Technol Assess 1999;3:1-156.

20. Edwards W. The theory of decision making. Psychol Bull 1954;51:380-417.

21. Rowe SL, French RS, Henderson C, et al. Help-seeking behaviour and adolescent self-harm: a systematic review. Aust $N Z$ J Psychiatry 2014:48:1083-95.

22. Annalisa@. Secondary. http://www.annalisa.org.uk

23. Elicia@. Secondary. http://www.maldaba.co.uk/products/elicia/

24. Dolan JG. Multi-criteria clinical decision support a primer on the use of multiple-criteria decision-making methods to promote evidence-based, patient-centered healthcare. Patient 2010;3:229-48.

25. French RS, Cowan FM, Wellings $\mathrm{K}$, et al. The development of a multi-criteria decision analysis aid to help with contraceptive choices: my contraception tool. J Fam Plann Reprod Health Care 2014;40:96-101.

26. O'Connor RC, Rasmussen S, Miles $\mathrm{J}$, et al. Self-harm in adolescents: self-report survey in schools in Scotland. $\mathrm{Br}$ J Psychiatry 2009;194:68-72.

27. Baetens I, Claes L, Muehlenkamp J, et al. Non-suicidal and suicidal self-injurious behavior among Flemish adolescents: a web-survey. Arch Suicide Res 2011;15:56-67.

28. Christensen $\mathrm{H}$, Griffiths KM, Jorm AF. Delivering interventions for depression by using the internet: randomised controlled trial. $B M J$ 2004;328:265-68A.

29. Jorm AF, Griffiths KM. Population promotion of informal self-help strategies for early intervention against depression and anxiety. Psychol Med 2006;36:3-6.

30. Griffiths KM, Christensen H, Jorm AF, et al. Effect of web-based depression literacy and cognitive-behavioural therapy interventions on stigmatising attitudes to depression: randomised controlled trial. Br J Psychiatry 2004;185:342-9.

31. Webb M, Burns J, Collin P. Providing online support for young people with mental health difficulties: challenges and opportunities explored. Early Interv Psychia 2008;2:108-13.

32. Esbensen FA, Melde C, Taylor TJ, et al. Active parental consent in school-based research: how much is enough and how do we get it? Evaluation Rev 2008;32:335-62 